\title{
GENERALIZED FIRST BOUNDARY VALUE PROBLEM FOR SCHRÖDINGER EQUATION
}

\author{
REN YANXIA
}

(Communicated by Barbara L. Keyfitz)

\begin{abstract}
In this paper, we have obtained two main results by using probabilistic methods: (i) For a domain, we obtained a representation formula of the bounded solution to the first boundary value problem for Schrödinger equation; (ii) For $\alpha \in R^{1}$, under certain conditions, we proved that the bounded solution having limit $\alpha$ at infinity to the generalized first boundary value problem for Schrödinger equation exists and is unique, and it is represented in explicit formula. The results of this paper are generalizations of Chung and Rao.
\end{abstract}

\section{INTRODUCTION}

In $1954 \mathrm{~J}$. L. Doob gave an intensive study of Laplace's equation on a domain $D$ from a probabilistic point of view. The bounded solution to the generalized Dirichlet problem for Laplace's equation has an interpretation in terms of Brownian motion (see [7]). Recently, many authors have been interested in the probabilistic treatment of the following Schrödinger equation:

$$
\frac{1}{2} \Delta u+c u=0 \text { in } D .
$$

When $m(D)<\infty$, Chung and Rao [2] solved the problem of representing the bounded solution to the first boundary value problem for Schrödinger equation. But when $m(D)=\infty$, can we solve the first boundary value problem for Schrödinger equation probabilistically? It seems that this work has not been explored up to now. This paper contributes to the answer of such a question to some extent.

Let $R^{d} \quad(d \geq 1)$ be a $d$-dimensional Euclidean space. A domain $D$ in $R^{d}$ is an open connected set; its boundary is $\partial D=\bar{D} \cap \overline{D^{c}}$, where $\bar{D}$ is the closure and $D^{c}$ the complement of $D$. Let $\{B(t), t \geq 0\}$ be the standard Brownian motion in $R^{d} . P_{x}$ and $E_{x}$ denote the probability and expectation for the Brownian motion starting at $x$. The class of bounded Borel measurable functions in $D$ will be denoted by $\mathrm{bB}(D)$.

For any Borel set $E$ we put

$$
\tau_{E}=\inf \{t>0, B(t) \notin E\} ;
$$

Received by the editors July 6, 1990 and, in revised form, January 22, 1991.

1991 Mathematics Subject Classification. Primary 60J45. 
namely, the first exit time from $E$, with the usual convention that inf $\phi=$ $\infty$. The class of points that is regular for $E$ will be denoted by $E^{r}, E^{r}=$ $\left\{x: P_{x}\left(\tau_{E}=0\right)=1\right\}$ (see [7]). Let $\tau=\tau_{D}$. For $c \in \mathrm{bB}(D)$, as an abbreviation we put

$$
\left.\left.c_{t}=\int_{0}^{t} c(B(r)) d y, \quad c_{s, t}=\int_{s}^{t} c(B)\right\rangle\right) d r .
$$

We say that $f$ is Hölder continuous in $D$, iff for any compact subset $C$ of $D$ there exist two constants $\alpha>0$ and $M$ such that $|f(x)-f(y)| \leq M\|x-y\|^{\alpha}$ for $x$ and $y$ in $C$, where $\|\cdot\|$ is the Euclidean norm in $R^{d}$.

If $c$ is a bounded, integrable and Hölder continuous function in $D$ and $\varphi$ is an essentially bounded and essentially continuous function on $\partial D$ (see the definition in [7]), the purpose of this paper is to consider the following generalized first boundary value problem for the Schrödinger equation:

$$
\begin{cases}\frac{1}{2} \Delta u+c u=0, \quad \text { in } D, & \\ \lim _{D \ni x \rightarrow b} u(x)=\varphi(b), & b \in \partial D \cap\left(D^{c}\right)^{r} \text { and } b \text { is a point at which } \varphi \\ & \text { is continuous }\end{cases}
$$

(where $\Delta$ is the Laplace operator, i.e., $\Delta=\sum_{i=1}^{d} \partial^{2} / \partial x_{i}^{2}, x=\left(x_{1}, x_{2}, \ldots, x_{d}\right)$ $\left.\in R^{d}\right)$.

We know, if $\varphi$ is an essentially bounded and essentially continuous function on $\partial D$, then $f$ is the bounded solution to the following problem (2) iff there exists $\alpha \in R^{1}$ such that $f=E .(\varphi(B(\tau)) ; \tau<\infty)+\alpha P .(\tau=\infty)$ on $D$.

$$
\begin{cases}\frac{1}{2} \Delta u=0, \quad \text { in } D, & \\ \lim _{D \ni x \rightarrow b} u(x)=\varphi(b), & b \in \partial D \cap\left(D^{c}\right)^{r} \text { and } b \text { is a point at which } \varphi \\ & \text { is continuous. }\end{cases}
$$

Under certain conditions, $E .(\varphi(B(\tau)) ; \tau<\infty)+\alpha P .(\tau=\infty)$ is the unique bounded solution to problem (2) having limit $\alpha$ at infinity (see [7]). In this paper, we will obtain similar results to problem (1).

\section{SOME LEMMAS}

Lemma 1. Let $c \in \mathrm{bB}(D)$.

(i) If $E .\left(\exp \left(c_{\tau}\right) ; \tau=\infty\right) \not \equiv \infty$ in $D$, then it is bounded on every compact subset of $D$.

(ii) For a measurable function $\varphi$ on $\partial D$, if $E .\left(|\varphi|(B(\tau)) \exp \left(c_{\tau}\right) ; \tau<\infty\right) \not \equiv$ $\infty$ in $D$, then it is bounded on every compact subset of $D$.

Proof. (ii) is the result of Theorem 1.1 in [2]. The proof of (i) is similar to that of Theorem 1.1 in [2].

Lemma 2. Let $d \geq 3$ and $c$ be a bounded and integrable function on $D$. Then $E .\left(\int_{0}^{\tau} c(B(t)) d t\right)$ is bounded on $D$. If in addition $D$ is unbounded, then

$$
\lim _{\substack{x \in D \\\|x\| \rightarrow \infty}} E_{x}\left(\int_{0}^{\tau} c(B(t)) d t\right)=0 .
$$


Proof. For $x \in D$, we have

$$
\begin{aligned}
E_{x}\left(\int_{0}^{\tau} c(B(t)) d t\right) & \leq E_{x}\left(\int_{0}^{\infty}|\tilde{c}|(B(t)) d t\right) \\
& =\frac{\Gamma(d / 2-1)}{2 \pi^{d / 2}} \int_{D} \frac{|c(y)|}{\|x-y\|^{d-2}} d y,
\end{aligned}
$$

where

$$
\tilde{c}(y)= \begin{cases}c(y), & y \in D \\ 0, & y \notin D\end{cases}
$$

By using analytic methods, we can show that

$$
\int_{D} \frac{|c(y)|}{\|x-y\|^{d-2}} d y
$$

is bounded on $D$ and

$$
\lim _{\substack{x \in D \\\|x\| \rightarrow \infty}} \int_{D} \frac{|c(y)|}{\|x-y\|^{d-2}} d y=0
$$

if $D$ is unbounded. Hence, the desired conclusion follows from these observations.

Lemma 3. Suppose $d \geq 3$. Let $\varphi$ be an essentially bounded and essentially continuous function on $\partial D$ and, if $D$ is unbounded, let $\varphi$ have limit $\alpha$ at infinity, where $\alpha \in R^{1}$. Then $E .(\varphi(B(\tau)) ; \tau<\infty)+\alpha P .(\tau=\infty)$ is the unique bounded solution to the following generalized Dirichlet problem:

$$
\begin{cases}\frac{1}{2} \Delta u=0, & \text { on } D ; \\ \lim _{D \ni x \rightarrow b} u(x)=\varphi(b), & b \in \partial D \cap\left(D^{c}\right)^{r} \text { and } b \text { is a point at which } \varphi \\ \lim _{x \in D,\|x\| \rightarrow \infty} u(x)=\alpha, & \text { is continuous; } \\ \text { is } \text { inbounded. }\end{cases}
$$

Proof. When $D^{c}$ is transient, the result follows from Theorem 4.2.11 in [7]. When $D^{c}$ is recurrent the result also follows by using Theorem 4.2.10 in [7] together with the conclusion that $\{B(t), t \geq 0\}$ is a recurrent process if $d \geq 3$.

\section{MAIN RESUltS}

Theorem 1. Let $c$ be bounded and Hölder continuous on $D$ and $\varphi$ be a measurable function on $\partial D$. If

$$
u_{1}=E .\left(\exp \left(c_{\tau}\right) ; \tau=\infty\right) \not \equiv \infty
$$

and

then $u_{1}$ and $u_{2}$ satisfy the equation

$$
u_{2}=E \cdot\left(|\varphi|(B(\tau)) \exp \left(c_{\tau}\right) ; \tau<\infty\right) \not \equiv \infty \quad \text { on } D,
$$

$$
\frac{1}{2} \Delta u+c u=0 \text { on } D .
$$

Proof. Let $D_{0}$ be any bounded subset of $D$ such that $\bar{D}_{0} \subset D$. We have by the strong Markov property,

$$
u_{1}=E .\left(\exp \left(c_{\tau_{D_{0}}}\right) u_{1}\left(B\left(\tau_{D_{0}}\right)\right) \text { on } D_{0}\right.
$$


and

$$
u_{2}=E \cdot\left(\exp \left(c_{\tau_{D_{0}}}\right) u_{2}\left(B\left(\tau_{D_{0}}\right)\right) \text { on } D_{0}\right. \text {. }
$$

Now, $u_{1}$ and $u_{2}$ are bounded by Lemma 1 . Using the method similar to that of Theorem 2.1 in [2], we can prove $u_{1}$ and $u_{2}$ really satisfy

$$
\frac{1}{2} \Delta u+c u=0 \text { on } D \text {. }
$$

Theorem 2. Let $c$ be a bounded and Hölder continuous function on $D$ and $E .\left(\exp \left(c_{\tau}\right)\right)$ be bounded on $D$. Then for any essentially bounded and essentially continuous function $\varphi$ on $\partial D$, we have $E .\left(\varphi(B(\tau)) \exp \left(c_{\tau}\right) ; \tau<\infty\right)+$ $\alpha E .\left(\exp \left(c_{\tau}\right) ; \tau=\infty\right)$ is a bounded solution of equation (1), where $\alpha \in R^{1}$.

Proof. From Theorem $1 E .\left(\varphi(B(\tau)) \exp \left(c_{\tau}\right) ; \tau<\infty\right)+\alpha E .\left(\exp \left(c_{\tau}\right) ; \tau=\infty\right)$ is a bounded solution of the equation

$$
\frac{1}{2} \Delta u+c u=0 \text { on } D \text {. }
$$

By the method of proving Theorem 1.3 in [2], we can show that $u_{2}=$ $E .\left(\varphi(B(\tau)) \exp \left(c_{\tau}\right) ; \tau<\infty\right)$ satisfies

$$
\begin{array}{ll}
\lim _{D \ni x \rightarrow b} u_{2}(x)=\varphi(b), & b \in \partial D \cap\left(D^{c}\right)^{r} \text { and } b \text { is a point } \\
& \text { at which } \varphi \text { is continuous, }
\end{array}
$$

and $u_{1}=E \cdot\left(\exp \left(c_{\tau}\right) ; \tau=\infty\right)$ satisfies

$$
\lim _{D \ni x \rightarrow b} u_{1}(x)=0, \quad b \in \partial D \cap\left(D^{c}\right)^{r} .
$$

So the desired conclusion follows from the above observations.

Let $c, f \in \mathrm{bB}(D)$. If $D$ is Greenian, define

$$
G_{D}^{c} f=E .\left(\int_{0}^{\tau} \exp \left(-c_{t}\right) f(B(t)) d t\right) ; \quad G_{D}^{0} f=G_{D} f \text { on } D .
$$

Theorem 3. Suppose $d \geq 3$. Let $c$ be a bounded, integrable, and Hölder continuous function on $D$, and let $\varphi$ be an essentially bounded and essentially continuous function on $\partial D$. If $E .\left(\exp \left(c_{\tau}\right)\right)$ is bounded on $D$, then $u$ is $a$ bounded solution to equation (1) if and only if there is an $\alpha \in R^{1}$ such that

$$
u=E .\left(\varphi(B(\tau)) \exp \left(c_{\tau}\right) ; \tau<\infty\right)+\alpha E .\left(\exp \left(c_{\tau}\right) ; \tau=\infty\right) \text { on } D \text {. }
$$

Suppose $d \leq 2$. If $D$ is Greenian and $E .\left(\int_{0}^{\tau} c(B(t)) d t\right)$ is bounded on $D$, under the above conditions, we have the same result.

Proof. Let $d \geq 3$. If $u$ is a bounded solution of (1), then

$$
v=u-E \cdot\left(\varphi(B(\tau)) \exp \left(c_{\tau}\right) ; \tau<\infty\right) \text { on } D
$$

is a bounded solution of (1) in which $\varphi \equiv 0$. Since $G_{D}|c|$ is bounded on $D$ by Lemma 2 , so that $\left|G_{D}(c v)\right| \leq\|v\|_{\infty} G_{D}|c|$ is bounded on $D$ (where $\|v\|_{\infty}=$ $\left.\sup _{x \in D}|v(x)|\right)$ and

$$
\begin{gathered}
\lim _{\substack{x \in D \\
x \rightarrow b}} G_{D}|c|(x)=0, \quad b \in \partial D \cap\left(D^{c}\right)^{r} \quad(\text { see }[7, \text { Theorem 4.6.7]), } \\
G_{D}(c v)=-2 c v \quad(\text { see }[7, \text { Theorem 4.6.6]). }
\end{gathered}
$$


These imply that $v-G_{D}(c v)$ is a bounded solution of (2) in which $\varphi \equiv 0$. Therefore we have that there is an $\alpha \in R^{1}$ such that

$$
v-G_{D}(c v)=\alpha P .(\tau=\infty) \quad(v \text { is bounded }) \text { on } D .
$$

Let $\rho(x)=|c(x)|+c(x), x \in D$. It is obvious that $\rho(x) \geq 0, x \in D$. Using the Markov property, it is easy to check that

$$
\begin{aligned}
G_{D}(c v) & =G_{D}^{|c|}\left(|c| G_{D}(c v)\right)+G_{D}^{|c|}(c v) ; \\
P .(\tau=\infty) & =E .\left(\exp \left(-|c|_{\tau}\right) ; \tau=\infty\right)+G_{D}^{|c|}(|c| P .(\tau=\infty)) \quad \text { on } D,
\end{aligned}
$$

so we have

$$
v=G_{D}^{|c|}(\rho v)+\alpha E \cdot\left(\exp \left(-|c|_{\tau}\right) ; \tau=\infty\right) .
$$

Let $M$ be the operator of multiplication by $\rho$. By successive substitution from the above equality we find

$$
\begin{aligned}
v= & \alpha E \cdot\left(\exp \left(-|c|_{\tau}\right)\left(\sum_{k=0}^{n}\left(\int_{0}^{\tau} \rho(B(t)) d t\right)^{k} / k !\right) ; \tau=\infty\right) \\
& +\left(G_{D}^{|c|} M\right)^{n+1} v \text { on } D,
\end{aligned}
$$

for any positive integer $n$. Now by hypothesis, $E .\left(\exp \left(c_{\tau}\right)\right)<\infty$, in $D$. Then we have

$$
\begin{gathered}
E .\left(\exp \left(c_{\tau}\right)\right)=E .\left(\exp \left(-|c|_{\tau}\right)\left(\sum_{k=0}^{\infty}\left(\int_{0}^{\tau} \rho(B(t)) d t\right)^{k} / k !\right)\right)<\infty \\
E .\left(\exp \left(c_{\tau}\right)\right)=\sum_{k=0}^{\infty}\left(G_{D}^{|c|} M\right)^{k} E .\left(\exp \left(-|c|_{\tau}\right)\right)<\infty \quad \text { on } D .
\end{gathered}
$$

Since $E .\left(\exp \left(-|c|_{\tau}\right)\right) \geq \exp \left(-E .\left(|c|_{\tau}\right)\right)$ by Jensen's inequality applied to the convex function $e^{-t}$, we have

$$
\inf _{D} E .\left(\exp \left(-|c|_{\tau}\right)\right)>0
$$

Let $n \rightarrow \infty$ in (4), it follows from (5), (6), and (7) that

$$
v=\alpha E .\left(\exp \left(c_{\tau}\right) ; \tau=\infty\right) \text { in } D .
$$

Thus when $d \geq 3$ the theorem is true.

When $d \leq 2$ it can be proved similarly.

Theorem 4. Suppose $d \geq 3$. Let $c$ be a bounded, integrable, and Hölder continuous function on $D$ such that $E .\left(\exp \left(c_{\tau}\right)\right)$ is bounded on $D, \varphi$ be an essentially bounded and essentially continuous function on $\partial D$ and, if $D$ is unbounded, $\lim _{x \in \partial D,\|x\| \rightarrow \infty} \varphi(x)=\alpha \in R^{1}$. Then

$$
u=E .\left(\varphi(B(\tau)) \exp \left(c_{\tau}\right) ; \tau<\infty\right)+\alpha E .\left(\exp \left(c_{\tau}\right) ; \tau=\infty\right)
$$

is a bounded solution to the following problem:

$$
\begin{cases}\frac{1}{2} \Delta u+c u=0, & \text { on } D \\ \lim _{D \ni x \rightarrow b} u(x)=\varphi(b), & b \in \partial D \cap\left(D^{c}\right)^{r} \text { and } b \text { is a point at which } \varphi \\ & \text { is continuous, } \\ \lim _{\|x\| D} u(x)=\alpha, & \text { if } D \text { is unbounded. }\end{cases}
$$


Proof. To prove the theorem, we only need to prove that if $D$ is unbounded, then

$$
\lim _{\substack{x \in D \\\|x\| \rightarrow \infty}} u(x)=\alpha
$$

( $u$ satisfies ( $8 \mathrm{a})$ and $(8 \mathrm{~b})$ by Theorem 2 ).

By Markov property, we have

$$
\begin{aligned}
G_{D}(c u)= & E \cdot\left(\int_{0}^{\tau} c(B(t)) E_{B(t)}\left(\varphi(B(\tau)) \exp \left(c_{\tau}\right) ; \tau<\infty\right) d t\right) \\
& +\alpha E \cdot\left(\int_{0}^{\tau} c(B(t)) E_{B(t)}\left(\exp \left(c_{\tau}\right) ; \tau=\infty\right) d t\right) \\
= & E .\left(\varphi(B(\tau)) \int_{0}^{\tau} c(B(t)) \exp \left(c_{t, \tau}\right) d t ; \tau<\infty\right) \\
& +\alpha E \cdot\left(\int_{0}^{\tau} c(B(t)) \exp \left(c_{t}, \tau\right) d t ; \tau=\infty\right) \\
= & E .\left(\varphi(B(\tau)) \exp \left(c_{\tau}\right)-\varphi(B(\tau)) ; \tau<\infty\right) \\
& +\alpha E \cdot\left(\exp \left(c_{\tau}\right)-1 ; \tau=\infty\right) \\
= & u-E .(\varphi(B(\tau)) ; \tau<\infty)-\alpha P .(\tau=\infty) .
\end{aligned}
$$

Hence,

$$
u-G_{D}(c u)=E \cdot(\varphi(B(\tau)) ; \tau<\infty)+\alpha P .(\tau=\infty) \text { on } D .
$$

Let $u_{0}=E .(\varphi(B(\tau)) ; \tau<\infty)+\alpha P .(\tau=\infty)$ on $D$. It follows from Lemmas 2 and 3 that

$$
\lim _{\substack{x \in D \\\|x\| \rightarrow \infty}} u_{0}(x)=\alpha ; \quad \lim _{\substack{x \in D \\\|x\| \rightarrow \infty}} G_{D}(c u)=0 .
$$

Thus $\lim _{x \in D,\|x\| \rightarrow \infty} u(x)=\alpha$.

If we let $\varphi=\alpha=1$ in Theorem 4, we can easily have

Corollary 1. Suppose $d \geq 3$ and $D$ is unbounded. Let $c$ be a bounded, integrable, and Hölder continuous function on $D$. If $E .\left(\exp \left(c_{\tau}\right)\right)$ is bounded on $D$, then

$$
\lim _{\substack{x \in D \\\|x\| \rightarrow \infty}} E_{x}\left(\exp \left(c_{\tau}\right)\right)=1 .
$$

Theorem 5. Suppose $d \geq 3$. Let $c$ be a bounded, integrable, and Hölder continuous function on $D, \varphi$ be an essentially bounded and essentially continuous function on $\partial D$, and, if $D$ is unbounded, $\lim _{x \in \partial D,\|x\| \rightarrow \infty} \varphi(x)=\alpha \in R^{1}$. Then the bounded solution to (8) is unique.

Proof. To prove Theorem 5, we only need to prove if $\varphi \equiv 0$ and $\alpha=0$, problem (4) has only the solution $u \equiv 0$.

If $u$ is a bounded solution to (8) in which $\varphi \equiv 0, \alpha=0$, then $u-G_{D}(c u)$ is a bounded solution to (3) in which $\varphi \equiv 0, \alpha=0$. It follows from Lemma 3 that

$$
u-G_{D}(c u)=0 .
$$

By the proof of Theorem 3, we know $u \equiv 0$.

Summing up the results of Theorems 4 and 5, we conclude with a corollary. 
Corollary 2. Under the conditions of Theorem 4,

$$
u=E .\left(\varphi(B(\tau)) \exp \left(c_{\tau}\right) ; \tau<\infty\right)+\alpha E .\left(\exp \left(c_{\tau}\right) ; \tau=\infty\right)
$$

is the unique bounded solution to (8).

\section{FURTHER RESULTS}

Now we consider the following statements:

(a) $E .\left(\exp \left(c_{\tau}\right)\right) \not \equiv \infty$ in $D$;

(b) $E .\left(\exp \left(c_{\tau}\right)\right)<\infty$ in $D$;

(c) $E .\left(\exp \left(c_{\tau}\right)\right)$ is bounded in $D$.

We know, if $m(D)<\infty$, then (a) and (c) are equivalent. For any domain $D$, we only know (a) and (b) are equivalent. But we do not know if (a) and (c) are equivalent. The following result can possibly give some relations between (a) and (c).

Theorem 6. Let $d \geq 3$ and $c$ be a bounded, integrable, and Hölder continuous function such that $E .\left(\exp \left(c_{\tau}\right)\right) \not \equiv \infty$ in $D$. If there exists an essentially bounded and essentially continuous function $\varphi_{0}$ on $\partial D$ that satisfies

1. $\lim _{x \in \partial D,\|x\| \rightarrow \infty} \varphi_{0}(x)=\alpha_{0} \in R^{+}$, if $D$ is unbounded;

2. $\inf _{x \in \partial D} \varphi_{0}(x)>0$;

3. problem (8) in which $\varphi=\varphi_{0}$ has a bounded solution, then $E .\left(\exp \left(c_{\tau}\right)\right)$ is bounded in $D$.

Proof. Let $u$ be a bounded solution to problem (8) in which $\varphi$ is an essentially bounded and essentially continuous function on $\partial D$, and if $D$ is unbounded, $\lim _{x \in \partial D,\|x\| \rightarrow \infty} \varphi(x)=\alpha \in R^{1}$. We will show that

$$
u=E .\left(\varphi(B(\tau)) \exp \left(c_{\tau}\right) ; \tau<\infty\right)+\alpha E .\left(\exp \left(c_{\tau}\right) ; \tau=\infty\right) \text { in } D .
$$

By Lemma 2 and Theorems 4.6.6, 4.6.7 in [7], we know

$$
u-E \cdot\left(\int_{0}^{\tau} c(B(t)) u(B(t)) d t\right)
$$

is a bounded solution to (3). It follows from Lemma 3 that

$$
u-E .\left(\int_{0}^{\tau} c(B(t)) u(B(t)) d t\right)=E .(\varphi(B(\tau)) ; \tau<\infty)+\alpha P .(\tau=\infty) \text { in } D .
$$

For simplicity, let us introduce some notations. Let $\alpha \in R^{1}, f$ and $c$ be bounded measurable functions on $D, c \geq 0$ and $\varphi$ be an essentially bounded measurable function on $\partial D$. We put

$$
\begin{gathered}
E .\left(\int_{0}^{\tau} \exp \left(-c_{t}\right) f(B(t)) d t ; \tau<\infty\right)=\widetilde{G}_{D}^{c} f ; \\
E .\left(\varphi(B(\tau)) \exp \left(-c_{\tau}\right) ; \tau<\infty\right)=H_{D}^{c} \varphi ; \\
E .\left(\int_{0}^{\tau} \exp \left(-c_{t}\right) f(B(t)) d t ; \tau=\infty\right)=\bar{G}_{D}^{c} f ; \\
E .\left(\alpha \exp \left(-c_{\tau}\right) ; \tau=\infty\right)=\bar{H}_{D}^{c} \alpha ; \\
\widetilde{G}_{D}^{0} f=\widetilde{G}_{D} f ; \quad H_{D}^{0} \varphi=H_{D} \varphi ; \quad \bar{G}_{D}^{0} f=\bar{G}_{D} f ; \quad \bar{H}_{D}^{0} \alpha=\bar{H}_{D} \alpha \quad \text { in } D .
\end{gathered}
$$


Thus we have

$$
u=\widetilde{G}_{D}(c u)+H_{D} \varphi+\bar{H}_{D} \alpha+\bar{G}_{D}(c u) \text { in } D .
$$

Let $\rho$ be defined as in Theorem 3. For a bounded measurable function $f$ on $D$, we can prove that

$$
\begin{gathered}
H_{D} \varphi=H_{D}^{|c|} \varphi+\widetilde{G}_{D}^{|c|}\left(|c| H_{D} \varphi\right) \quad \text { in } D ; \\
\widetilde{G}_{D}(c u)=\widetilde{G}_{D}^{|c|}\left(|c| \widetilde{G}_{D}(c u)\right)+\widetilde{G}_{D}^{|c|}(c u) \quad \text { in } D ; \\
\bar{H}_{D} \alpha=\bar{H}_{D}^{|c|} \alpha+\bar{G}_{D}^{|c|}\left(|c| \bar{H}_{D} \alpha\right) \quad \text { in } D ; \\
\bar{G}_{D}(c u)=\bar{G}_{D}^{|c|}\left(|c| \bar{G}_{D}(c u)\right)+\bar{G}_{D}^{|c|}(c u) \quad \text { in } D ; \\
\widetilde{G}_{D}^{|c|}\left(f\left(\bar{H}_{D} \alpha\right)+f \bar{G}_{D}(c u)\right)=0 \text { in } D ; \\
\bar{G}_{D}^{|c|}\left(f H_{D} \varphi+f \widetilde{G}_{D}(c u)\right)=0 \text { in } D .
\end{gathered}
$$

It follows from (9) and the above equalities that

$$
u=H_{D}^{|c|} \varphi+\widetilde{G}_{D}^{|c|}((|c|+c) u)+\bar{H}_{D}^{|c|} \alpha+\bar{G}_{D}^{|c|}((|c|+c) u) \text { in } D .
$$

So we have changed (9) to another form

$$
u=H_{D}^{|c|} \varphi+\widetilde{G}_{D}^{|c|}(\rho u)+\bar{H}_{D}^{|c|} \alpha+\bar{G}_{D}^{|c|}(\rho u) \text { in } D .
$$

By the method of successive iteration, we can obtain for any positive integer $n$,

$$
\begin{aligned}
u= & E \cdot\left(\varphi(B(\tau)) \exp \left(-|c|_{\tau}\right)\left(\sum_{k=0}^{n}\left(\int_{0}^{\tau} \rho(B(t)) d t\right)^{k} / k !\right) ; \tau<\infty\right) \\
& +E \cdot\left(\alpha \exp \left(-|c|_{\tau}\right)\left(\sum_{k=0}^{n}\left(\int_{0}^{\tau} \rho(B(t)) d t\right)^{k} / k !\right) ; \tau=\infty\right) \\
& +\left(G_{D}^{|c|} M\right)^{n+1} u \quad(M \text { is defined as in Theorem 3) in } D .
\end{aligned}
$$

Let $n \rightarrow \infty$ in (17). It follows from (5), (6), (7) that

$$
u=E .\left(\varphi(B(\tau)) \exp \left(c_{\tau}\right) ; \tau<\infty\right)+\alpha E .\left(\exp \left(c_{\tau}\right) ; \tau=\infty\right) \text { in } D .
$$

$u$ is bounded in $D$.

In particular, if $\varphi=\varphi_{0}$, we know $E .\left(\exp \left(c_{\tau}\right)\right)$ is bounded in $D$.

\section{REFERENCES}

1. M. Aizenman and B. Simon, Brownian motion and Harnack's inequality for Schrödinger operators, Comm. Pure Appl. Math. 35 (1982), 209-273.

2. K. L. Chung, Notes on the inhomogeneous Schrödinger equation, Seminar on Stochastic Processes 1984, Birkhauser, Boston, 1986.

3. K. L. Chung and K. M. Rao, Feynman-Kac functional and the Schrödinger equation, Seminar on Stochastic Processes, Birkhauser, Boston, 1981.

4. R. Durrett, Brownian motion and martingales in analysis, Wadsworth, Belmont, CA, 1984.

5. N. Falkner, Feynman-Kac function and positive solutions of $\frac{1}{2} \Delta u+q u=0, \mathrm{Z}$. Wahrsch. Verw. Gebiete 65 (1983), 19-33.

6. Z. C. Li, The extension of the Dirichlet problem, Acta Math. Sci. 4 (1984), 237-296. 
7. S. C. Port and C. J. Stone, Brownian motion and classical potential theory, Academic Press, New York, 1978.

8. B. Simon, Schrödinger semigroups, Bull. Amer. Math. Soc. 7 (1982), 447-526.

Hebei Institute of Mechanical Electrical Engineering, Shijiazhuang, People's RepubLIC OF CHINA 